\title{
Reaction path and reactive transport modeling of rare earth elements: Insights into the evolution of fractionation patterns
}

\author{
KAREN H. JohanNESSON ${ }^{1 *}$, Christopher D. White ${ }^{1}$, \\ AND SEGUN B. ADEBAYO ${ }^{2}$ \\ ${ }^{1}$ School for the Environment, University of Massachusetts \\ Boston, Boston, MA, USA, karen.johannesson@umb.edu \\ (*presenting author) \\ ${ }^{2}$ Department of Earth and Environmental Sciences, Tulane \\ University, New Orleans, LA, USA
}

Interest in the rare earth elements (REE) is on the rise because of their importance to the "green economy". Specifically, REEs are used in manufacturing mobile phones, hybrid automobiles, magnets, and in wind power generation, among others. As such, these critical metals are likely candidates for future emerging environmental contaminants. Indeed, application of gadolinium diethylenetriaminepentaacetic acid (Gd-DPTA), as a magnetic contrast agent for medical imaging has introduced orders of magnitude higher Gd concentrations to certain environments. The REEs are also chemical analogs of the radioactive actinide series elements, and thus can be used to provide a first order grasp of actinide fate and transport in the environment. Although aqueous complexation models for REEs are relatively robust, surface complexation models are more limited. Both are necessary to simulate the fate and transport of the highly reactive REEs in the environment. Here, we couple the one-dimensional advective-dispersive flow equation to an aqueous and surface complexation model for the REEs in a well-studied, confined aquifer from Texas, USA, to gain insights into the important processes that control the transport of REEs in groundwaters. The model also provides an understanding of how reactive transport of REEs impacts their fractionation patterns along flow paths as the composition of the groundwater evolves owing to biogeochemical reactions occurring within the aquifer. We also employ reaction path modeling to investigate REE fractionation pattern evolution in a stratified, "meromictic" estuary. Here, mixing of river water, submarine groundwater discharge, and coastal seawater, along with adsorption onto precipitating Mn oxides in the oxic surface waters followed by reductive dissolution of these oxides in the anoxic waters can explain the observed changes in REE fractionation patterns within the estuary. 